\title{
Des guerriers pour faire la paix. L'armée américaine en Irak
}

\section{Thomas Lindemann}

\section{(2) OpenEdition \\ Journals}

Édition électronique

URL : http://journals.openedition.org/conflits/3114

DOI : $10.4000 /$ conflits.3114

ISSN : $1777-5345$

Éditeur :

CCLS - Centre d'études sur les conflits lilberté et sécurité, L'Harmattan

Édition imprimée

Date de publication : 15 novembre 2007

Pagination : 13-34

ISBN : 978-2-296-04582-8

ISSN : 1157-996X

Référence électronique

Thomas Lindemann, «Des guerriers pour faire la paix. L'armée américaine en Irak », Cultures \& Conflits

[En ligne], 67 | automne 2007, mis en ligne le 04 janvier 2010, consulté le 30 mars 2021. URL : http:// journals.openedition.org/conflits/3114; DOI : https://doi.org/10.4000/conflits.3114 


\section{Des guerriers pour faire la paix. L'armée américaine en Irak}

\section{Thomas LINDEMANN}

Thomas Lindemann est professeur de science politique à l'université Montesquieu Bordeaux-IV. Il a notamment co-dirigé avec M.-L. Martin Les Militaires et le recours à la force armée, Paris, L'Harmattan, 2006 et publié "Faire la guerre, mais laquelle? Les institutions des Etats-Unis entre identités bureaucratiques et préférences stratégiques ", Revue française de science politique, octobre 2003 ; Les Doctrines darwiniennes et la guerre de 14, Paris, Economica, 2001. Il publiera prochainement un ouvrage sur les origines de la guerre (La Guerre pour la reconnaissance, à paraître début 2008).

$\mathrm{L}$ orsque l'armée américaine prend, le 9 avril 2003, le contrôle de Bagdad, rien ne laisse présager qu'elle deviendra l'ennemi de la population irakienne. Si les troupes américaines ne reçoivent pas l'accueil triomphal espéré, leur présence est pour autant saluée par de nombreux Irakiens dans l'espoir d'éviter le retour du régime baasiste. Ils sont convaincus que leur « sort ne peut que s'améliorer après des années de souffrance 1 ». Quatre ans plus tard, le bilan de l'action américaine semble pour le moins mitigé : des milliers de soldats ont été tués, les attentats contre les forces armées américaines et le régime en place sont devenus quotidiens ${ }^{2}$. En outre, des cas de torture et de mauvais traitements mettant en cause des soldats américains sont apparus au grand jour. Même les observateurs les plus optimistes n'imaginent pas que les violences civiles pourraient rapidement cesser.

Le plus souvent, les difficultés des troupes américaines en Irak sont ramenées au contexte international et interne. Certaines renvoient au manque de légitimité du recours à la force américaine contre l'Irak, fondé à l'origine sur la prétendue existence des armes de destruction massive. D'autres insistent sur la composition hétérogène de la population irakienne associée à des rancunes historiques et à la lutte pour la domination entre populations sunnites et chiites. Ces facteurs auraient rendu l'éclosion des violences civiles plus ou moins

1. Lafourcade F., Le Chaos irakien, Paris, La Découverte, 2007, p. 46.

2. Keegan J., The Iraq War. The 21-Day Conflict and its Aftermath, Londres, Pimlico, 2005. 
inévitable. Toutefois, l'intensité d'une violence civile est variable. On peut difficilement écarter l'hypothèse selon laquelle les « bévues » des armées américaines dans leurs opérations de maintien de l'ordre ont joué un rôle non négligeable dans l'escalade des violences civiles en Irak ${ }^{3}$.

Le propos de notre article est d'examiner sous des facettes multiples la question de l'aptitude de l'armée américaine aux missions de maintien de l'ordre en Irak. Quel est le bilan sécuritaire de l'armée américaine en Irak par rapport aux autres armées, comme celle de la Grande-Bretagne par exemple ? Quelles sont les raisons des dérapages sécuritaires des forces américaines ? Les difficultés tiennent-elles plus à la culture organisationnelle des armées américaines ou bien à une incompétence plus générale des forces armées dans les missions de maintien de l'ordre?

\section{La variable dépendante : l'adéquation des armées aux missions de maintien de l'ordre}

Contrairement à des opérations de guerre classique, l'objectif d'une mission de maintien de l'ordre et de « contre-insurrection ${ }^{4}$ dans un pays lointain consiste moins à abattre l'ennemi qu'à " gagner les cours de la population». Le peuple est, selon Mao, «le grand océan dans lequel l'ennemi se noiera 5 ". Cet objectif suppose des comportements stratégiques modérés et empathiques. Premièrement, les forces de maintien de l'ordre doivent soigneusement doser l'usage de la force en évitant les «dommages collatéraux » susceptibles de dresser les populations civiles contre la force militaire occupante. Plus concrètement, toute violation des règles d'engagement constitue un usage disproportionné de la force. L'expression « règles d'engagement » (RE) désigne les directives régissant l'emploi de la force armée par les soldats dans un théâtre d'opérations. Ainsi, les règles d'engagement dans les démocraties occidentales interdisent généralement aux soldats d'ouvrir le feu lorsque leur vie n'est pas en danger. Tirer sur des lanceurs de pierres voire sur de simples manifestants et, plus généralement, tout acte de violence gratuit ( $\mathrm{y}$ compris la torture) constituent donc des usages disproportionnés de la force.

On peut aussi évaluer l'adéquation d'une armée à des missions de maintien de l'ordre par sa volonté d'assumer des tâches plus civiles, comme la maintenance

3. Voir à ce propos Cohen E.A., "L'armée américaine après l'Irak », Critique internationale, $\mathrm{n}^{\circ} 31$, avril-juin 2006, pp. 9-21, particulièrement p. 12 et suivantes.

4 . Bulloch G., "Military doctrine and counter-insurgency. A British perspective," Parameters, n²6, été, 1996 ; Mockaitis T.R., British Counter-Insurgency, 1919-60, New York, St. Martin’s Press, 1990 ; Cohen E.A., "Constraints on America's conduct of small wars", International Security, automne 1984, pp. 172-173 ; Mack A., "The concept of power and its uses in explaining asymmetric conflict”, London, Richardson Institute for Conflict and Peace Research, 1997 ; Greene T.N., The Guerrilla and How to Fight Him, New York, Praeger, 1962, pp. 1415 ; Hart L., The British Way in Warfare, New York, The Macmillan Company, 1933, p. 97.

5 . Mao Zedong, La Guerre révolutionnaire, Paris, Editions sociales, 1951. 
des autoroutes, la distribution de la nourriture et de l'eau, voire l'enseignement. La participation d'une armée à l'aide concrète des populations en détresse est susceptible de leur démontrer leur caractère « amical ». La distribution des paquets Care ${ }^{6}$ dans l'Allemagne de l'après-guerre a beaucoup contribué à forger l'image de l'«ami » américain, incarné par le GI noir qui distribue du chocolat aux enfants ${ }^{7}$. En revanche, le cantonnement des soldats d'occupation à des fonctions strictement sécuritaires peut être interprété comme la preuve qu'il s'agit bien de forces « étrangères », de soldats insensibles aux besoins réels de la population.

Enfin, une armée engagée dans une opération de maintien de l'ordre doit tenir compte des particularités culturelles du pays « hôte » si elle ne veut pas s'aliéner la population. Par exemple, les forces armées respectent-t-elles les lieux saints ou le statut de la femme et des personnes âgées ? La communication entre forces armées et populations civiles est liée au respect des cultures. Existe-t-il des interprètes et quels sont les gestes non verbaux des forces armées envers les civils?

Selon nous, de la réponse à ces questions dépend en grande partie la capacité de l'armée occupante à se légitimer auprès des populations locales et à isoler les groupes armés résistants. Contrairement à des perspectives « fixistes ", nous estimons que l'hostilité irakienne envers les troupes américaines n'était pas inscrite dans le marbre. En réalité, l'animosité irakienne a constitué une sorte de prophétie auto-réalisatrice : les soldats américains, en traitant les Irakiens comme des ennemis potentiels, ont contribué à ce qu'ils se perçoivent comme radicalement autres. Les sondages d'opinion effectués en Irak indiquent nettement que le rejet des «forces de l'occupation » n'intervient qu'après une série de «bavures » des soldats américains : attitude passive de l'armée américaine face aux pillages qui ravagent les villes au lendemain de sa « victoire », bombardement indiscriminé de la ville de Falloudjah par les corps de marines en avril 2004 ou encore, révélation des actes de torture dans la prison d'Abou Ghraib. Ainsi, selon un sondage de $\mathrm{BBC}, \mathrm{ABC}$ NEWS, NHK auprès d'un échantillon représentatif de 2000 Irakiens entre 2004 et 2007, près de $60 \%$ des interrogés estimaient les attaques contre les forces américaines justifiées ${ }^{8}$. De même, alors qu'en 2004 encore $49 \%$ des interrogés (13\% de non-réponses) estimaient encore l'intervention américaine en Irak au printemps 2003 justifiée ou partiellement justifiée, seulement $37 \%$ des interrogés exprimaient cette même opinion en août 2007 9. Un rapport d'Amnesty International de juillet 2005 résume ainsi cette évolution :

«Les Irakiens ont, de manière générale, accueilli favorablement la chute de Saddam Hussein, mais l'opposition à la présence de la force

6. Care: Cooperative for Assistance and Relief Everywhere. Il s'agit de ravitaillements de nourriture et autres denrées par l'association humanitaire Care International.

7. Voir Ziemke E.F., U.S. Army in the Occupation of Germany 1944-46, United States Governement Printing, 1983.

8. BBC, ABC News et NHK opinion poll on Iraq, 10 septembre 2007, oraqananalysis.org

9. Ibid. 
multinationale est répandue... Les Irakiens sont profondément irrités par l'effondrement des institutions nationales et les dommages causés à leur héritage culturel, notamment le pillage et l'incendie des universités, lycées et musées 10 ».

En d'autres termes, s'interroger sur l'adéquation des comportements stratégiques des forces armées à leur mission de maintien de l'ordre n'est pas un luxe. Au contraire, l'intensité de la violence civile dépend en grande partie des interactions de l'armée avec la population et du degré de mépris ou de reconnaissance qu'elle exprime à son égard.

\section{Pour une approche constructiviste « matérialiste »}

Comment comprendre l'adéquation plus ou moins forte des armées aux opérations de maintien de l'ordre ? Notre analyse tente de combiner une approche "constructiviste » mettant en avant les spécificités identitaires dans la définition des «intérêts » des armées américaines avec l'approche « bureaucratique » postulant l'existence d'intérêts corporatistes stables dans toute institution sociale, comme le désir d'obtenir un minimum de ressources, d'autonomie et de « reconnaissance » pour sa « survie organisationnelle » 11 .

De l'approche constructiviste ${ }^{12}$, nous retenons d'abord l'idée que les identités ne sont pas exogènes aux interactions sociales. Elles se forment notamment par rapport aux « autrui significatifs 13 ». Cela signifie que les identités dépendent en grande partie de ce que les autres en font. Des identifications positives entre forces armées et populations civiles sont possibles grâce à des comportements coopératifs répétés. Ainsi, la pertinence de notre interrogation sur le comportement des troupes américaines en Irak est fondée sur

10. «Irak. Des atteintes aux droits humains perpétrées de sang-froid par des groupes armés », Amnesty International, document public, Index AI : MDE 14/009/2005, Londres, juillet 2005.

11. Voir pour l'approche théorique : Snyder J., The Ideology of the Offensive: Military Decision Making and the Disasters of 1914, Ithaca, Cornell University Press, 1984, pp. 24-34 et Posen B., The Sources of Military Doctrine, Ithaca, Cornell University Press, 1984, p. 47 et suivantes; Byman D.L., Waxman M.C., "Kosovo and the great air power debate", International Security, vol. $24, \mathrm{n}^{\circ} 4$, printemps 2000 , pp. 5-38 ; Mitchell P.T., “Ideas, interests, and strategy: bureaucratic politics and the United States Navy", Armed Forces and Society, vol. 25, n², Winter 1999, pp. 243-265 ; Vennesson P., Les Chevaliers de l'air. Aviation et conflits au XXe siècle, Paris, Sciences Po, 1997 ; Lindemann T., "Faire la guerre, mais laquelle ? Les institutions militaires des Etats-Unis entre identités bureaucratiques et préférences stratégiques ", Revue française de science politique, octobre 2003, pp. 675-706.

12. Voir par exemple Wendt A., Social Theory of International Politics, Cambridge University Press, 1999 ; P. Katzenstein (ed.), The Culture of National Security, New York, Columbia University Press, 1996 (en particulier le chap. 2 : Jepperson R.L., Wendt A., Katzenstein P., "Norms, identity, and culture in national security", pp. 33-75) ; Kier E., "Culture and military doctrine: France between the wars”, International Security, vol. 19, n4, printemps 1995, pp. 65-93 ; Johnston A.I., “Thinking about military culture”, International Security, vol. 19, $\mathrm{n}^{\circ} 4$, printemps 1995, pp. 32-64.

13. Voir Mead G.H., L'Esprit, le soi et la société, Paris, PUF, 1963 (traduction de Mind, Self and Society, 1934). 
la prémisse que les identités sont en mutation constante. En effet, si la violence civile n'était qu'une question d'opportunité, comme le suggèrent les thèses d'inspiration réaliste, toute tentative de construire des identifications positives, voire des identités partagées via des comportements de reconnaissance, serait vouée à l'échec. De même, plus la force est employée de manière dissuasive voire brutale par les forces de l'ordre et plus les insurgés devraient être enclins à capituler. Or, les événements en Irak démontrent au contraire que l'intensification des bombardements, loin de conduire à une pacification, entraine une radicalisation des groupes armés et même des populations civiles.

Toutefois, le fait que les identités sont susceptibles de changer ne signifie pas qu'elles sont totalement perméables. Quant aux cultures organisationnelles des forces armées américaine et britannique, nous considérons qu'elles se sont si fortement institutionnalisées qu'elles sont difficilement modifiables. Ainsi, aux EtatsUnis, l'identité du « soldat combattant » s'est solidement enracinée depuis la guerre du Vietnam via des enseignements dans les écoles militaires, les récits des aînés et même l'entraînement physique privilégiant la manipulation des armes lourdes. Cet ethos du combat est devenu quasiment « naturel » pour les militaires américains.

\section{La première variable explicative : l'identité organisationnelle}

Nous empruntons à l'approche constructiviste une deuxième idée selon laquelle les normes et les identités constituent les intérêts des acteurs. Pour paraphraser Alexander Wendt, les institutions militaires ne peuvent pas savoir ce qu'elles veulent sans savoir qui elles sont ou qui elles veulent être ${ }^{14}$. De ce fait, il importe d'investir les normes et les identités des organisations militaires afin de comprendre leur intérêt à recourir à la force armée et à préconiser des options offensives en vue d'une « victoire décisive » sur l'ennemi. Un soldat qui cultive sa différence avec la société civile et l'ethos combattant aura un plus grand «intérêt » à riposter « brutalement » face à des provocations mineures qu'un soldat qui se perçoit lui-même davantage comme membre de la société civile. Un présupposé implicite des interprétations constructivistes est l'hypothèse selon laquelle les acteurs n'ont pas simplement des intérêts matériels, mais aussi un intérêt symbolique à confirmer leur « identité ». Cet intérêt dépend à son tour de coûts psychologiques et même matériels lorsque l'image de soi et les identités revendiquées ne sont pas confirmées par autrui. Ainsi, un militaire avec un référentiel identitaire hérö̈que aura une image dépréciative de soi s’il est jugé inapte par ses supérieurs à participer à des missions de combat. Selon nous, le manque de préparation de l'armée américaine aux missions de maintien de l'ordre est bel et bien un produit de son identité organisationnelle depuis la guerre du Vietnam, reposant sur l'exaltation d'un «professionnalisme radical 15 » hostile aux missions

14. Voir à ce propos Wendt A., Social Theory of International Relations, op. cit., chap. IV.

15. Voir à ce propos Caplow T., Vennesson P., Sociologie militaire, Paris, Armand Colin, 2000 ; Boëne B., (dir.), La Spécificité militaire, Paris, Armand Colin, 1990. 
plus « civiles ». Cette spécificité « culturelle » de l'armée américaine est une cause primordiale de son inadéquation aux missions de maintien de l'ordre. La réponse aux questions suivantes permettra l'identification empirique du référentiel identitaire d'une armée : les militaires insistent-ils sur leur «spécificité » en mettant l'accent sur leur fonction spécifiquement «guerrière » (le "professionnalisme radical ») 16 ? Ou bien adhèrent-ils à une conception sécularisée voire banalisée de leur métier en admettant le pluralisme (militaire, diplomatique, etc.) ?

\section{La seconde variable explicative : les spécificités militaires}

Toutefois, contrairement à ce que préconiserait une approche constructiviste radicale, nous estimons qu'une fois constituée, une organisation aura des intérêts stables, y compris matériels Une fois créée, chaque organisation a des besoins fondamentaux pour sa survie que l'on qualifiera d'intérêts stables. On peut raisonnablement émettre l'hypothèse que les institutions militaires fortement différenciées possèdent des intérêts « minimaux » en termes de ressources, de prestige et d'autonomie. Cette vision est partagée par l'approche bureaucratique qui part de la prémisse selon laquelle toute organisation sociale possède des intérêts corporatistes. Ainsi, une armée mal équipée comme l'armée russe durant la Première Guerre mondiale court le danger d'une désintégration rapide. De même, une armée dépourvue de toute autonomie et soumise à des purges constantes comme l'Armée rouge de Staline serait menacée d'inefficacité opérationnelle. Toujours est-il qu'une armée dépréciée par l'opinion interne risque de subir de nombreuses défections, comme l'institution militaire américaine durant la guerre du Vietnam. Mais toute armée tient surtout sa raison d'être de ses compétences « combatives ». L'apprentissage des opérations de maintien de l'ordre par les armées se heurte donc également aux spécificités institutionnelles de la profession militaire. Même les armées les plus «civilianisées 17 » cherchent à préserver un noyau irréductible : celui de leur mission combative. Chaque armée - y compris celles qui ont un ethos professionnel «banalisé »- est entraînée pour mener et gagner des guerres. C’est cette exigence «fonctionnelle» du conditionnement combatif qui éclaire pour nous une autre partie des dérapages sécuritaires lors des opérations de maintien de l'ordre, comme en Irak. Ainsi, nous allons démontrer que ce sont plus particulièrement les unités spécialisées dans les combats comme les marines qui ont tendance à un usage disproportionné de la force.

16. Janowitz M., The Professional Soldier, New York-Londres, The Free Press, 1971 (rééd.), p. 21 ; Huntington S., The Soldier and the State: The Theory and Politics of Civil-Military Relations, Cambridge Mass., Harvard University Press, 1957, p. 61 ; Moskos C., "Institutional and occupational trends in armed forces”, in Moskos C., Wood F., (eds), The Military: More than just a job?, Washington/New York, Pergamon-Brassey's, 1988, p. 16 ; Boene B., "Permanence et relativité de la spécificité militaire. Examen critique de la littérature existante, esquisse d'une synthèse », in Boëne B. (dir.), op. cit., pp. 188-240.

17 . Voir Joana J., «Les politiques de ressource humaine des armées en France et en GrandeBretagne », Revue française de science politique, vol. 54, n 5 , pp. 811-827. 
Selon nous, les perspectives constructiviste et bureaucratique sont compatibles. La dernière vient éclairer les intérêts stables d'une organisation militaire qui sont indissociablement liés à sa survie. Au sens d'Alexander Wendt, on peut dire que l'entraînement combatif est constitutif de l'existence même des forces armées. Il fait donc partie de leur « identité de corps » ( corporate identity»). En revanche, l'approche constructiviste éclaire les intérêts plus variables d'une institution militaire, comme par exemple la mission à laquelle elle aspire ("role identity»). Il existe une très grande variété d'options stratégiques pour satisfaire les intérêts « corporatistes » de l'institution militaire. Rien ne détermine par exemple a priori si une armée doit privilégier les opérations de combat classique par rapport aux opérations d'une plus faible intensité, comme celles du maintien de l'ordre, pour obtenir plus de prestige ou de ressources. Cet intérêt dépendra en grande partie des matrices normatives et identitaires des institutions militaires comme l'existence d'un professionnalisme plus ou moins radical.

Afin d'examiner les référentiels identitaires et les spécificités combatives des armées, nous nous référons autant que possible aux documents produits par les acteurs eux-mêmes, comme les instructions de combat, les doctrines stratégiques, les maquettes publicitaires ou encore leurs devises et chansons. En outre, il existe désormais une littérature secondaire non négligeable à ce sujet. Quant à l'analyse des comportements des armées américaine et britannique en Irak, nous nous appuyons sur des informations provenant de sources diverses aussi bien officielles (les enquêtes internes des armées) que journalistiques, ou encore, celles provenant d'acteurs non gouvernementaux comme les enquêtes réalisées par Amnesty International. Le caractère encore «chaud " de ces informations doit naturellement nous inciter à la prudence. Autant que possible, nous tenterons de mobiliser des sources diverses afin de vérifier les témoignages des acteurs sur le terrain.

Dans un premier temps, nous démontrerons que les armées américaines étaient peu disposées à déclencher une guerre contre l'Irak en raison de leur crainte d'être impliquées par la suite dans une longue mission pacificatrice. Cette mission civile suscite beaucoup plus de réserves auprès des forces armées américaines que les missions classiques de combat. Ensuite, nous examinerons les pratiques stratégiques de l'armée américaine dans sa mission de maintien de l'ordre. Selon nous, elle a commis de multiples bavures depuis quatre ans en Irak. Une comparaison avec les forces britanniques à Bassorah permettra d'objectiver les inadéquations de l'armée américaine dans sa mission de maintien de l'ordre. Dans une deuxième partie, il importe de comprendre les raisons des variations stratégiques entre les forces américaines et britanniques. Nous nous attachons à identifier les déterminants « identitaires » et «bureaucratiques» dans la stratégie de pacification américaine. D’une part, nous établirons un lien entre identités organisationnelles et usage disproportionné de la force armée en comparant l'identité organisationnelle de l'armée 
britannique ${ }^{18}$, plus ouverte aux missions civiles, avec celle de l'armée américaine cultivant l'hérö̈sme. D'autre part, nous allons démontrer que ce sont plus particulièrement les unités «combatives » telles que les marines qui sont enclines à l'escalade lors des opérations de maintien de l'ordre. Plus généralement, l'entraînement guerrier des armées avec ses routines organisationnelles de combat est peu compatible avec les opérations de peacekeeping. En d'autres termes, nous établirons des «variations concomitantes » entre, d'un côté, le référentiel hérö̈que d'une culture organisationnelle ainsi que les spécificités militaires et, d'un autre côté, l'inadéquation aux opérations de maintien de l'ordre qui se traduit par un usage disproportionné de la force.

\section{Un usage difficilement approuvé et disproportionné de la force armée}

\section{L'acceptation hésitante de la guerre contre l'Irak par l'armée américaine}

Le rôle des militaires dans la décision de recourir à la force contre l'Irak en 2003 a été modeste. Les grandes délibérations dans le « cabinet de guerre » du 21 décembre 2002 ou du 25 janvier 2003 du président Bush semblent avoir été menées sans que les forces armées soient intervenues de manière décisive ${ }^{19}$. Le responsable du Central Command (Centcom), Tommy Francks, à qui incombait en cas de guerre la responsabilité de la conduite des opérations, exprima à plusieurs reprises ses inquiétudes au président Bush. Des réserves ont également été formulées par les chefs des quatre armées de la Navy, des marines, de l'Air Force et de l'Army 20. Les responsables de l'armée de terre étaient en particulier préoccupés par la perspective d'une occupation prolongée de l'Irak. D’ailleurs, la résistance la plus forte contre le déclenchement de la guerre en Irak à l'intérieur de l'administration Bush provint du secrétaire d'Etat Colin Powell, l'ancien chef d'état-major conjoint des armées. Une telle opposition fut encore plus franchement affichée par d'anciens responsables militaires, comme les généraux Schwarzkopf et Zinni. Commandant en chef des opérations armées durant la guerre du Golfe, le général Schwarzkopf remarque à propos du secrétaire à la Défense Donald Rumsfeld : "Certains de ses propos m'ont inquiété. [...] Dans ses commentaires, il paraît qu'il ne tient pas compte de l'armée ». Derrière ses réserves transparaît la crainte d'enlisement de l'armée en Irak : "A quoi ressemblera l'Irak après la guerre, avec les Kurdes, les Sunnites et les Chiites? C'est une question primordiale, à mon avis. Cela devrait faire partie du plan de campagne général 21 ». Quant au général Anthony Zinni, ancien chef du commandement central de l'armée

18. Voir à ce propos Woodward B., Plan d'attaque, Paris, Pocket, 2004, p. 369

19. Laurent E., La Guerre des Bush, Paris, Pocket, 2003, p. 139 et suivantes.

20. Voir à ce propos Moskos C., The American Enlisted Man, New York, Russell Sage, 1970. Voir aussi Boëne B., Dandecker C., (dirs), Les Armées en Europe, Paris, La Découverte, Paris, 1998 et Martin M.L., From Warriors to Managers, Chapel Hill, 1981.

21. Cité par solidariteetprogres.online.fr/News/Etats-Unis/breve_660.html 
américaine, il a déclaré dans une interview publiée dans Nerwsweek le 3 février 2003 :

«Au départ, on pouvait au moins insinuer que l'Irak était lié au terrorisme... Maintenant, on en est réduit à dire : "Vous ne nous laissez pas parler avec vos scientifiques”, pour justifier la guerre. $\mathrm{Ou}$ encore: "Nous savons ce que possèdent les Irakiens, mais nous ne pouvons pas vous le dire.” Tout ceci est vraiment trop confus ».

La question du volume de l'effectif engagé en Irak constituait un autre clivage majeur entre forces armées et pouvoir civil. Alors que Donald Rumsfeld militait en faveur d'une force d'intervention réduite et modernisée de 105000 hommes, l'état-major se prononça en faveur d'une force près de cinq fois plus importante ${ }^{22}$. En octobre 2003, lors d'une réunion entre le président et les quatre chefs des armées, le chef d'état-major de l'armée de terre, Eric Shinseki, dénonça le fait que les unités prévues pour l'offensive n'étaient pas assez nombreuses. Durant son mandat de chef d'état-major de l'US Army, Eric Shinseki a eu de nombreuses altercations publiques avec le secrétaire à la Défense. Donald Rumsfeld, champion de la Révolution dans les affaires militaires et de la "rationalisation ", accusa à de multiples reprises les militaires de vouloir se battre avec un état d'esprit d'autrefois. Ce conflit atteignit son apogée lors du témoignage d'Eric Shinseki devant la Commission des forces armées du Sénat à propos des effectifs nécessaires à une «bonne occupation » de l'Irak. Le général James Jones, le commandant en chef des marines, partageait également les inquiétudes du général Shinseki sur la suffisance de l'effectif engagé. En outre, il s'interrogea sur la capacité de l'armée américaine à faire face à l'éventualité d'une attaque chimique.

Si l'armée américaine hésitait à s'engager dans une telle guerre, c'est principalement en raison de la mission de maintien de l'ordre qui se profilait à l'horizon de l'après-guerre. Les oppositions plus ou moins feutrées des responsables des armées américaines à l'égard des interventions en Somalie (1992-1993), en Bosnie (1995) et même au Kosovo (1999), avaient aussi pour motivation la crainte d'engager l'armée dans des opérations « coûteuses » en vies humaines, ne rapportant aucune " gloire » militaire. L'intervention Restore Hope en Somalie (1992-1993) constitua un tournant, au sens où l'attitude sceptique des militaires américains envers les opérations de maintien de la paix se transforma en franche hostilité, après avoir subi des pertes dans une opération de poursuite du général Aideed. En 1997, l'ancien chef d'état-major Shalikashvili rappela que, face à la multiplication de leurs missions, il était «important de mettre en avant la compétence centrale des forces armées, qui est la suivante : le combat 23 ».

22. Cohen E.A., op. cit., p. 12 et suivantes.

23. Shinseki E.A., "Adress to the Eisenhower Luncheon”, $45^{\text {th }}$ meeting of the association of the United States Army, 12 october :

www.army.mi/armyvision/senior_99eisenhower_speech.htm 
Toutefois, au fur et à mesure de l'installation des forces armées américaines en Arabie saoudite et ailleurs, les responsables militaires furent probablement de plus en plus disposés à s'engager dans une confrontation armée pour des raisons «techniques». Après des déploiements échelonnés de 10000 , 15000 et 20000 hommes, l'armée américaine atteignit en février 2003 un effectif d'environ 140000 hommes dans la région du Golfe. Très rapidement, les militaires rappelèrent aux décideurs politiques la difficulté de maintenir longtemps en «attente » une force si imposante, pour des raisons logistiques et même psychologiques. Une autre difficulté résidait dans la disposition de la Turquie et de l'Arabie saoudite à mettre «durablement » à disposition américaine leur territoire pour le stationnement des troupes. Lors de son entretien avec le président Bush le 9 janvier 2003, le général Francks remarque que "dans la mesure où les déploiements avaient commencé depuis quelques temps, ils avaient déjà brûlé un certain nombre d'étapes 24 ». Enfin les responsables militaires ne voulaient pas à " patienter » car l'armée irakienne pourrait alors renforcer son dispositif défensif et, ainsi, mieux anticiper et contrer leur plan d'attaque. Lors d'une rencontre le 11 janvier 2003 à la Maison blanche avec Richard Cheney et Donald Rumsfeld, Richard Myers, le chef de l'état-major interarmées exprimait ses regrets d'avoir "perdu l'effet stratégique de surprise après nous être enlisés dans le processus des Nations unies et nous sommes en train de perdre l'effet tactique de surprise... 25 ».

En somme, l'armée américaine était donc initialement plutôt réservée envers l'emploi de la force armée contre l'Irak. Les missions de maintien de l'ordre qui se profilaient à l'horizon de la guerre classique étaient l'une des raisons de cette hésitation.

\section{Les difficultés dans les missions de maintien de l'ordre}

\section{Le comportement stratégique de l'armée américaine}

La guerre contre l'Irak ne dura que quelques semaines et confirma d'abord le point de vue moderniste du Pentagone et de son chef, qui s'étaient prononcés pour le high-tech war, engageant des effectifs relativement restreints. Toutefois, plus de trois ans après le début de la guerre, tout porte à croire que l'armée américaine a commis des erreurs nombreuses et importantes dans sa mission «pacificatrice » en Irak.

Tout d'abord, l'armée américaine communique peu avec la population irakienne. Le nombre d'interprètes américains est minime. Les Irakiens sont de cette façon souvent contrôlés sans communication «verbale " véritable. Même durant leurs "loisirs ", les soldats américains n'ont quasiment aucun

24. Woodward B., op. cit., p. 384.

25. Ibid., p. 395. 
contact avec la population. Alors que les forces armées scandinaves sont réputées pour leur conduite civile dans les opérations de maintien de la paix jouant par exemple en Macédoine au volley-ball pendant les missions d'observation -, les soldats américains sont lourdement armés et ont l'interdiction formelle d'adresser la parole aux populations civiles ${ }^{26}$. Dans la revue Quadriennal, le document du Pentagone précisant la posture des forces armées pour une durée de quatre ans, constate à propos des soldats stationnés en Irak: "Ils vivent dans des camps fortifiés et éloignés de la population. La plupart de leurs contacts face-à-face avec les Irakiens s'établissent... durant les barrages, les fouilles ou les points de contrôle des vébicules 27 ». Ce manque de communication entre armée et population explique en partie le fait que les soldats sont plus perçus comme des occupants que comme des libérateurs. Mais ce manque de contact humain influence aussi l'attitude des soldats américains. Il n'est pas rare de les entendre dire que le seul moyen de communiquer avec les Irakiens est l'étalage de la «force».

Deuxièmement, les particularités culturelles de la population irakienne sont peu respectées. Certains soldats américains se promènent même torse nu lors des enterrements. Le destin du «monument aux morts » consacré aux martyres de la guerre de huit ans entre l'Iran et l'Irak à Bagdad est une illustration de ce manque d'empathie culturelle. Depuis l'arrivée des troupes américaines, un barbelé et un poste de sécurité américain rendent l'accès à ce lieu de recueillement quasi impossible pour les Irakiens. Pire encore, des ordures et des bouteilles de bière s'entassent tout près du monument. Les noms des victimes gravés sur le mur sont partiellement devenus illisibles en raison de l'affichage d'annonces accrochées par des soldats américains ${ }^{28}$. Il est très rare que les soldats aient reçu une formation de base sur l'histoire arabe et irakienne et sur leurs aspects culturels ${ }^{29}$. Un général américain affirme même qu'il serait irresponsable d'attendre d'un soldat du front qu'il risque et donne sa vie et qu'il développe en même temps "un niveau élevé de subtilité 30 ». Une femme irakienne qui s'était opposée à la politique de Saddam Hussein explique l'hostilité grandissante des populations irakiennes envers « l'occupant américain » ainsi :

«La plus grande erreur des forces d'occupation a été d'avoir agi au mépris de nos traditions et de notre culture. Ils ne se contentent pas d'avoir bombardé nos infrastructures, ils cherchent à détruire notre

26. Miller L., Moskos C., "Humanitarian warriors?”, Armed Forces and Society, vol. 21, n4, été 1995, pp. 615-638.

27. "They live in fortified camps away from the population and most face-to-face contact... is during cordon and search or vehicle checkpoint operations", DOD, Quadrennial Defense Review (QDR) 2006, vers. 3.1 (Washington, DC: U.S. Government Printing Office [GPO], 23 mai 2005).

28. Antoon S., "Monumental disrespect", Middle East Report, n²28, automne 2003, pp. 28-30.

29. Voir le témoignage d'un soldat de la $3 \mathrm{BCT}, 4^{\mathrm{e}}$ division d'infanterie : www.rokdrop.com/2007/03/24/useful-idiot-pshuman-kg

30. Cité par Aylwin-Forster B., op. cit, p. 5. 
système social et à atteindre notre dignité. En ça nous ne pouvons pas admettre... 31 ».

Troisièmement, les soldats américains ont fréquemment procédé à des contrôles de sécurité particulièrement irrespectueux des populations locales. Des cas ont été rapportés où des soldats ont infligé aux pères de famille un traitement humiliant devant leurs enfants. Dans d'autres occasions, ils ont défoncé des portes ou «profané » des symboles religieux - comme le Coran - ou des femmes, selon le «contexte intersubjectif » irakien. Ainsi près de Falloudjah, des soldats ont arraché à une femme une copie du Coran qu'elle tenait derrière son dos en la jetant par terre ${ }^{32}$. Cet incident déclencha une petite émeute dans la population locale. De toute évidence, la formation des soldats américains au contrôle des personnes civiles était rudimentaire. Souvent, ils appliquaient aux civils les procédures opérationnelles valables pour le contrôle des soldats ennemis, comme l'habitude de mettre un pied sur la tête de la personne, contrainte de s'allonger par terre.

Quatrièmement, l'armée américaine semblait peu équipée pour doser adéquatement l'usage de la force lors de ses opérations de maintien de l'ordre. Formellement, comme l'avons déjà vu, l'usage de la force ("rules of engagement ", règles de l'engagement) est restrictif et prévu pour des cas de légitime défense. Toutefois, l'entraînement militaire est axé sur le combat et l'anéantissement des forces adverses. Les stratégies contre - insurrectionnelles ont été très peu développées ${ }^{33}$. La revue Quadriennal de 2006 admet qu'il y avait au sein de l'armée une préférence pour «la mancuvre cinétique à grande échelle » et une focalisation sur l'élimination des insurgés et non sur la protection de la population ${ }^{34}$. Ce « culte » de l'offensive distingue les forces américaines des autres troupes. Les officiers américains reprochaient à leurs alliés britanniques et polonais d'être trop hésitants dans le recours à la force létale. Ils les accusèrent même d'encourager la résistance irakienne par leur manque de fermeté. Leurs réponses aux "provocations» ont été souvent disproportionnées. Un rapport d'Amnesty International de juillet 2005 précise :

«Le 23 avril 2003, des soldats américains sont arrivés à Falloudjah où ils ont occupé une école. Cinq jours plus tard, au moins 200 personnes ont manifesté contre l'utilisation de cette école comme caserne. Dixsept personnes ont été tuées et des dizaines d'autres blessées quand les soldats américains ont tiré sur la foule 35 ».

31. Rapport Amnesty International, op. cit.

32. CNN.com, "Bloody day in Iraq", novembre 2, 2003, interview avec Fawaz Gerges.

33. Voir à ce propos : Field Manual 100-8. The Army in Multinational Operations, 24 novembre 1997:

http://www.globalsecurity.org/military/library/policy/army/fm/dlmp_fm-inventory_v5_8.pdf et : www.loc.gouv./rr/frd/Military-Lawpdf/law-warhandbook-2005.pdf

34. "Preference for large-scale kinetic maneuver and focus on killing insurgents, not protecting the population", QDR, 2006, op. cit.

35. Rapport Amnesty International, juillet 2005, op. cit. 
Lorsqu’à Falloudjah, quatre "mercenaires » de la société de sécurité privée Blackwater ont été tués puis mutilés en avril 2004, la riposte a été immédiate. L’armée américaine décida de "nettoyer » la ville des insurgés. Les nombreux bombardements et « bévues » à l'occasion de cette opération radicalisèrent la population locale - un résultat certainement souhaité par les auteurs de cette "provocation 36 ». En novembre 2004, lors d'une autre opération de nettoyage, l'armée américaine tira quarante tours d'artillerie de calibre $155 \mathrm{~mm}$. Ce bombardement était considéré comme mineur par les responsables américains, si bien qu'il ne fut même pas rapporté par le commandant local du corps d'expédition de la marine au commandant central. Malgré l'expérience négative dans l'usage « intense » de la force, certains militaires américains estiment justement que l'armée est trop «scrupuleuse ». Des soldats d'infanterie et des sous-officiers de la $82^{\mathrm{e}}$ division aéroportée ont récemment publié un manifeste dans lequel ils militent pour un usage décomplexé de la force en Irak :

«Soyons clairs, nous avons la volonté et les moyens de combattre dans un tel contexte, mais nous sommes de facto paralysés parce que les réalités du terrain exigent des mesures auxquelles nous nous refusons toujours : le recours de façon massive, brutale et meurtrière à la force 37 ».

Enfin, des cas de torture ont été avérés dans la prison tristement célèbre d'Abou Ghraib. Le rapport officiel Fay-Jones de 2004 constate quarante cas d'abus de détenus dans cette prison. Ainsi, une compétition s'était engagée entre deux gardiens pour faire uriner des détenus à l'aide de leurs chiens. On rapporte des viols, et même des prisonniers battus à mort ${ }^{38}$. Pour certains soldats américains, l'ennemi n'était visiblement plus humain. Un déserteur de l'armée américaine estime même: "Aux yeux de l'armée, les Irakiens n'étaient pas des êtres bumains mais des terroristes, des kamikazes, des bougnoules du désert et de la racaille 39 ». Un capitaine admet avoir toujours inculqué à ses soldats que tuer n'est pas répréhensible si c'est pour l'idéal de la liberté 40 . La déshumanisation des Irakiens fut aussi favorisée par la rhétorique martiale du président Bush, insistant sur la menace que constituait un Irak nucléaire pour la liberté américaine.

\section{Le comportement stratégique de l'armée britannique}

Même s'il est difficile d'objectiver la "performance » de l'armée américaine, il semble que son bilan soit nettement plus négatif que celui des forces

36. Cité par Aylwin-Forster B., op. cit., p. 6

37. « Nous soldats américains en Irak », Le Monde, 28 août 2007.

38. Hooks G., Mosher C., "Outrages against personal dignity: rethinking abuse and torture in the war on terror", Social Forces, juin, vol. 83, n 4, pp. 1627-1646.

39. Key J., The Deserter's Tale, The Story of an Ordinary Soldier who walked away from War in Iraq, New York, Atlantic Monthly, 2007 (trad. française chez Albin Michel : Putain de guerre!).

40. Hooks G., Mosher C., op. cit. 
britanniques sur le terrain. L'officier britannique Brig Aylwin-Foster remarque dans son enquête sur l'armée américaine en Irak que celle-ci reste toujours majoritairement axée sur les opérations de combat, alors que l'armée britannique a intégré des stratégies de persuasion plus subtiles, visant à gagner «les cours et les esprits de la population » irakienne.

Tout d'abord les forces britanniques ont pris soin de rester en contact avec les populations locales. A Bassora, les responsables militaires ont associé des responsables religieux et des chefs de tribus à leurs décisions ${ }^{41}$. Si l'armée britannique prend aussi des mesures sécuritaires importantes, il n'en reste pas moins qu'elle utilise des patrouilles de routines à pied et, dans la mesure du possible, un armement léger pour établir des contacts avec la population civile. Elle patrouille avec des bérets et non des casques, et ses soldats ont l'instruction d'enlever leurs lunettes de soleil lorsqu'ils s'adressent à des populations locales. Les soldats britanniques s'affichent même la tête découverte face aux populations locales Ainsi, à la différence de l'armée américaine, les forces britanniques obtiennent des renseignements fondés sur des sources « humaines » (Human Intelligence, Humint) ${ }^{42}$. Un très grand nombre de soldats britanniques interviewés estiment ainsi que l'obsession américaine d'éviter les pertes est susceptible d'aliéner les populations irakiennes ${ }^{43}$. L'ancien commandant en chef des forces britanniques, Mike Jackson, a violemment critiqué en septembre 2007 l'orientation exclusivement « combative » de l'armée américaine en Irak. Donald Rumsfeld, à l'époque secrétaire d'Etat à la Défense, avait déclaré au lendemain de la victoire que les forces américaines n'étaient pas destinées au «nation building ». Or pour Jackson, cette attitude est symptomatique de la « faillite intellectuelle» de la politique militaire américaine ${ }^{44}$.

Des bévues sécuritaires ont aussi été commises par les forces britanniques fouillant par exemple avec des chiens des maisons à la recherche d'armes cachées. Or le chien est un animal «impur» dans l'imaginaire de l'Islam. Toutefois, selon un haut responsable militaire britannique, les militaires du Royaume-Uni étaient nettement moins optimistes que leurs collègues américains sur la perspective d'inculquer la démocratie « occidentale » aux Irakiens et plus sensibles au poids de l'histoire ${ }^{45}$.

Quant à l'usage excessif de la force, l'armée britannique habituée à des moyens militaires économiques plus limités, les emploie aussi avec plus de parcimonie en Irak. Contrairement à l'armée américaine, les forces britanni-

41. Webbe S., “How to beat Iraq's insurgents? Ask the British. Britain's knack for counterinsurgency is being ignored", The Christian Monitor, 23 juillet 2007.

42. Aylwin-Foster B., "Changing the army for counter insurgency operations", Military Review, nov-déc. 2005, pp. 5-15.

43. Garfield A., Bristish Perspectives on the U.S. Effort to Stabilize and Reconstruct Iraq, in early 2005, the Foreign Policy Research Institute (FPRI), 2006.

44 . Coughlin C., Tweedie N., "General Sir Mike Jackson attacks US over Iraq”, Daily Telegraph, 2 septembre 2007.

45 . Aylwin-Forster B., op. cit., p. 5. 
ques possèdent une longue expérience dans la stratégie de contre - insurrection que ce soit en Inde, en Palestine, en Malaisie ou en Irlande du Nord 46. En effet, par une stratégie de contre-insurrection efficace en Malaisie (19481960), l'armée britannique avait appris à doser savamment l'usage de la force pour ne pas s'aliéner les populations locales. Andrew Garfield estime ainsi que le principe d'une utilisation minimale de la force est inscrite dans la psyche de tous les officiers britanniques ${ }^{47}$. Si les comportements des forces britanniques sont loin d'être toujours «soft » envers les populations irakiennes, les fusillades « gratuites » restent le monopole des forces américaines.

La différence des approches américaine et britannique se traduit aussi dans le nombre de morts sur le terrain. Alors qu'environ 2,7 \% des soldats américains ont péri durant les quatre ans d'occupation (3 747 sur 140 000), 1,9\% des soldats britanniques (168 sur 8500 ) ont été tués durant cette période. Toutefois la réduction progressive des troupes britanniques de 30000 en avril 2003 à environ 7000 en octobre 2007, associée à l'image de la GrandeBretagne comme allié américain a plus récemment inversé cette tendance. Depuis un an, le taux de mortalité des soldats britanniques se rapproche de plus en plus de celui des soldats américains ${ }^{48}$.

Les «dénis de reconnaissance 49 » de l'armée américaine à l'égard de la population irakienne sont donc loin d'être anecdotiques. Ils apparaissent au contraire comme l'une des raisons de l'escalade de la violence. Il reste à vérifier si la nomination d'un spécialiste de contre-insurrection en janvier 2007 à la tête de l'armée américaine en Irak, David Petraeus, est susceptible d'améliorer la situation sécuritaire en Irak. Toutefois, les erreurs initiales de l'armée américaine constituent une hypothèque considérable pour une pacification durable. Comment donc expliquer ces divergences de performance dans les missions de maintien de l'ordre?

\section{Cultures militaires et corporatismes}

\section{Professionnalisme radical ou banalisation du métier des armes}

\section{Le professionnalisme radical de l'armée américaine}

La culture organisationnelle de l'armée américaine constitue une clef pour comprendre son insuffisante préparation à la mission « pacificatrice » en Irak. Dans l'ethos de l'armée américaine, cultivant l'image du « héros combattant », les

46. Cassidy R. M., "The British army and counterinsurgency. The salience of military culture", Military Review, mai-juin 2005, p. 53-59.

47. Ibid.

48. Hammer J., "Iraq. Basra breakdown”, Newsweek, 22 mai 2006.

49. Voir à ce propos Honneth A., Kampf um Anerkennung, Francfort a.M., Suhrkamp, 1992. 
opérations de maintien de la paix jouissent d'un faible prestige. Le nouveau commandant en chef des forces américaines en Irak, David H. Petraeus (2007), avoue dans la préface du manuel FM 3-24 consacré à la contre-insurrection que la plupart des décideurs politiques et militaires auraient négligé les opérations de «faible intensité » au profit de la guerre classique ${ }^{50}$. En effet, en 2000, l'ancien chef de l'état-major conjoint, Henry Shelton, refuse catégoriquement la formation d'une force spécialisée dans les opérations de maintien de la paix. Il estime que les «peacekeepers » les plus efficaces sont ceux qui possèdent des capacités dans les opérations de combat ${ }^{51}$. Le 11 avril 2003, le général Vincent Books, directeur adjoint des opérations des forces armées en Irak affirme qu' "à aucun moment nous ne voyons [les militaires américains] devenir véritablement une force policière. Ce que nous faisons c'est mener les actions [militaires] nécessaires pour créer les conditions de stabilité 52 ». D’ailleurs, l'impréparation des forces américaines aux missions civiles et au maintien de l'ordre explique en grande partie le chaos au lendemain de la victoire, caractérisé par des pillages systématiques, l'amassement des ordures ou le manque de distribution d'eau et d'électricité. A certains égards, on peut même constater une véritable aversion de l'institution militaire américaine à l'égard des opérations de maintien de l'ordre et de «nation-building ». Seul $1 \%$ environ des soldats participant aux opérations de maintien de la paix dans le monde est de nationalité américaine.

L’armée américaine cultive aussi un professionnalisme radical. Celui-ci se caractérise depuis la guerre du Vietnam par un « référentiel de repli » fondé sur une forte méfiance envers l'environnement civil, aussi bien la société que le pouvoir politique. Pour les officiers interrogés, la guerre du Vietnam a surtout été perdue sur le front «intérieur » en raison des agissements des décideurs politiques ${ }^{53}$. L'armée fustigea à l'époque les limites imposées par les dirigeants politiques, trop soucieux d'éviter l'escalade du conflit et "l'obligeant à se battre avec les mains derrière le dos ». Cette méfiance conduit les militaires à penser que les civils ne les soutiennent plus lorsqu'un conflit devient coûteux en vies humaines. Il faut rappeler que les plus hauts responsables de l'armée américaine d'aujourd'hui sont nés pour la plupart à la fin des années 1940 ou au début des années 1950. Ils ont donc presque tous participé à la guerre du Vietnam et le plus souvent intériorisé sa «prétendue » leçon sur la défaillance « civile » permettant de disculper l'armée. Entre-temps, la méfiance des militaires américains envers l'environnement civil s'est institutionnalisée à travers les enseignements dispensés dans les écoles de guerre, la transmission orale, les écrits des vétérans ou encore le culte des « soldats portés disparus ». Un cours dispensé à l'académie militaire de West Point, dans la rubrique « Military Art and Science » s'intitule

50. www.fas.org/irp/doddiv/army/fm3-24.pdf FM 3-24

51 . Henry Shelton, 2000 worldNETDayly.com

52. Cité par Brouse E.C., The US Army and Constabulary Operations, School of Advanced Military Studies, Fort Leavenworth, mai 2006, p. 54.

53 . Holsti O.R., Rosenau J.N., "The meaning of Vietnam: belief systems of American leaders", International Journal, vol. 32, n³, été 1977, pp. 452-474. 
«Korea, Vietnam and the American experience ». Le major Mc Master, qui a enseigné à West Point, a publié en 1997 un ouvrage intitulé de manière significative Derelection of Duty: Lyndon Johnson, Robert Mc Namara, The Joint Chiefs of Staff and the Lies that Led to Vietnam ${ }^{54}$. On peut raisonnablement faire l'hypothèse que cette «méfiance institutionnalisée » de l'armée américaine a incité ses responsables à rechercher une victoire « rapide » en Irak, par crainte d'enlisement dans le conflit et de la perte consécutive du soutien de l'opinion publique. Or, c'est précisément ce "culte de l'offensive ", cherchant à tout prix à anéantir les «terroristes ", qui provoque la résistance et la radicalisation de la population irakienne par les « dégâts collatéraux » qu'il engendre.

Deuxièmement, le référentiel de repli se caractérise par une conception exclusivement militaire du métier des armes. Cette vision dévalorise facilement les opérations de nature limitée où l'usage de la force est étroitement surveillé par le pouvoir civil et incompatible avec le désir d'obtenir la victoire sur le champ de bataille. L'identité plus étroitement militaire des hauts responsables se matérialise empiriquement par une valorisation des missions combattantes et un rejet des tâches plus civiles - comme les opérations de maintien de la paix -, une aversion à l'égard de l'usage modéré de la force, un mépris pour les intellectuels et l'exaltation de l'expérience guerrière.

Depuis la rupture constituée par le Vietnam, le modèle du soldat polyvalent («soldat-diplomate ») est devenu caduc. Cette guerre laissa à l'armée l'amère impression qu'elle avait été employée dans des missions pour lesquelles elle n'était pas faite. Le colonel Summers affirme ainsi que l'engagement politique de l'armée dans la lutte anti-guérilla était une erreur. Elle aurait dû se contenter de mener des opérations militaires classiques, en confiant à l'armée sud-vietnamienne la pacification des populations ${ }^{55}$. Depuis, un consensus s'est dégagé dans l'armée américaine : la mission du soldat est de servir et de préparer les grandes guerres de la nation ${ }^{56}$. Cette vision puriste conduisit même à des réserves à l'égard des missions mixtes comportant un volet «militaire » comme les opérations de maintien de la paix. Une enquête récente menée auprès des élèves de West Point démontre que les cadets de la première année ont encore une vision globalement positive des opérations de maintien de la paix. Mais cette approbation décline rapidement au fur et à mesure des années. Le pourcentage d'élèves présentant des symptômes de «l'esprit du combat » augmente, jusqu'à atteindre $65 \%$ en dernière année, alors qu'il n'est que de $39 \%$ à l'entrée de cette école 57 . L'esprit « combattant » était aussi fortement valorisé par le chef de l'armée de terre en fonction au début de la

54 . Mcmaster H.R., Derelection of Duty: Lyndon Johnson, Robert Mc Namara, The Joint Chiefs of Staff and the Lies that Led to Vietnam, New York, Harper Collins, 1997.

55. Summers H., On Strategy. A Critical Analysis of the Vietnam War, New York, Dell, 1984.

56 . Dunvin K.O., "Military culture: change and continuity", Armed Forces and Society, été 1994, pp. 531-547.

57. Franke V.C., "Warriors for peace: the next generation of US military leaders", Armed Forces and Society, automne 1997, pp. 33-57, p. 49. 
guerre contre l'Irak (2003), le général Shinseki. Il remarque en 1999 que « l'affaire principale de l'armée est de combattre et de gagner les guerres de la nation... Toutes nos missions sont subordonnées à cet objectif 58 ».

Il n'est pas sûr que l'armée américaine ait tiré les leçons de ses déboires. Si certains tentent d'adapter l'armée à des conflits où il ne s'agit plus de combattre des troupes ennemies mais de contrôler des territoires occupés par des civils ${ }^{59}$, d'autres au contraire estiment que l'armée devrait se recentrer sur ses missions combatives ${ }^{60}$. La réticence des hauts responsables envers le maintien de l'ordre pourrait même encore se renforcer si les pertes américaines augmentent à la suite d'embuscades tendues par les opposants à l'occupation américaine.

\section{Le professionnalisme pragmatique de l'armée britannique}

En revanche, l'armée britannique est nettement plus favorable aux missions plus civiles. Andrew Garfield note à propos de son enquête sur les soldats américains et britanniques en Irak :

«Un très grand nombre d'interviewés ont observé que les perceptions différentes des militaires britanniques et américains sur leur rôle peuvent indirectement conduire à une utilisation spécifique de la force létale. Les soldats américains se perçoivent eux-mêmes comme des guerriers dont le devoir est de finir avec les ennemis et de les détruire tandis que les soldats britanniques ont compris qu'ils peuvent faire la guerre aujourd'hui et le maintien de la paix demain. Cette différence entre "l'éthique du combattant" et "le maintien de l'ordre" aboutit à ce que le guerrier américain est trop facilement prédisposé à recourir à la force 61 ».

Certes, l'institution militaire britannique proclame elle aussi que sa mission est de "maintenir l'esprit combattant afin d'être efficace dans la guerre 62 ». Toutefois la «charte» des forces armées britanniques spécifie explicitement que les "opérations futures englobent le combat pour les perceptions, les cours et les idées » des populations ${ }^{63}$. Elle exige explicitement du soldat la formation de compétences autre que le combat et l'accomplissement de missions sur toute l'échelle de la violence («full spectrum»):

«l'absence d'un ennemi défini signifie que les soldats doivent s'abstenir de la réponse militaire la plus évidente...En complément aux

58. Shinseki E.K., "Adress to the Eisenhower luncheon”, 45 th Annual Meetining of the Association of the United States Army, 12 october : www.army.mil/armyvision/senior_99eisenhower_speech.htm

60 . Ricks T.E., "Lessons learned in Iraq show up in army class", Washington Post, 21 janvier 2006.

61. Voir à ce propos le «manifeste » des soldats américains dans Le Monde, op. cit.

62. Garfield A., op. cit.

63. The Military Covenant, chap. 2: www.army.mod.uk 
qualités militaires traditionnelles, cela implique une subtilité considérable et une maîtrise de soi dans tous les rangs...Les soldats de tous les rangs doivent être capables de confronter l'ambiguité, l'incertitude et le changement...Tout cela requiert une plus grande souplesse de tout soldat individuel $64 »$.

Le fait que la dimension combative de l'identité de l'armée britannique soit moindre s'explique notamment par sa «frugalité financière », ainsi que par son expérience en termes de «missions polyvalente» telles que le maintien de l'ordre en Inde, au Kenya, à Bornéo ou en Guyane. Très tôt, et surtout dans la période de l'entre-deux-guerres, les soldats britanniques ont donc du apprendre à économiser leurs munitions et à faire preuve d'une grande souplesse dans l'exécution de leurs tâches ${ }^{65}$. Ainsi en Malaisie (1948-1960), l'armée britannique combattait la guérilla communiste en organisant de petites patrouilles et en opérant dans les mêmes conditions que les insurgés et non avec l'artillerie lourde ou des forces aériennes. La pénétration clandestine des territoires ennemis, la sécurisation progressive des villages et la finesse dans l'emploi de la violence avaient plus d'importance dans la victoire que la machinerie lourde.

\section{La spécificité " combative » des militaires contre la " polyvalence »}

Mais les difficultés rencontrées par l'armée américaine sur le terrain irakien sont-elles toutes explicables par la «culture organisationnelle » de l'armée américaine ?

Un examen plus détaillé des auteurs des « dérapages » peut conduire à une vision plus nuancée. Ainsi, parmi la dizaine de cas jugés ou examinés par la justice militaire américaine en 2004 66, un nombre non négligeable d'auteurs ont « profité » d'un entraînement spécifiquement combattant alors que les « techniciens » de l'armée (les mécaniciens, les ingénieurs, informaticiens, spécialistes de la logistique, les administrateurs, et même la police militaire) ${ }^{67}$ sont nettement «sous-représentés » dans les excès de violence. Ainsi, deux des douze personnes suspectées d'abus dans les dix cas recensés appartiennent à la Navy SEALS, une unité de forces spéciales de nageurs de combat extrêmement polyvalents. Ils ont battu à mort un prisonnier dans la prison d'Abou Ghraib. Deux autres soldats, jugés pour le meurtre de trois Irakiens, appartiennent à la First Cavalry Division. Il s'agit d'une division blindée. Or de telles armes de mêlée bénéficient traditionnellement d'un prestige plus élevé que les armes d'appui et de soutien en raison de leur proximité au lieu de combat. Trois autres soldats, jugés pour

64. Ibid.

65 . Cassidy R.M., op. cit., pp. 53-59.

66. "Proceedings including possible offenses against protected persons in Iraq and Afghanistan", The American Journal of Law, janvier 2005, pp. 262-263.

67. Quant à la « banalisation» du métier des armes, voir Caplow T., Vennesson P., Sociologie militaire, op. cit., p. 18 et suivantes. 
avoir tué un Irakien gravement blessé, appartiennent à cette même division. Enfin, un autre «suspect », filmé à l'occasion d'une violence difficilement supportable contre un Irakien, appartient au corps des marines. Il est connu que ses membres cultivent un esprit « hérö̈que » et combatif. De l'officier général au cuisinier, en passant par le pilote de chasse, tous doivent savoir utiliser leur fusil et faire preuve d'une forme physique exemplaire. Parmi les douze personnes jugées, une seule fait partie de la police militaire et deux autres des services de renseignement. La plupart de ceux qui ont commis des actes de torture à Abou Ghraib sont des réservistes de l'armée de terre et ne sont donc pas membres du corps professionnel de la police militaire. L'ethos du combat transparaît même dans les justifications des accusés. Ainsi, Lynndie England, celle qui avait posé avec un Irakien tenu en laisse, explique à propos de la torture : "J'imagine que c'est toujours ce qui arrive en temps de guerre ». Elle prétend aussi ignorer la convention de Genève, qui protège les prisonniers de guerre. Malgré le caractère apologétique de ses déclarations, il fait peu de doutes que les unités combatives méprisent souvent les « civils » qui veulent appliquer le droit de la guerre et qui ont généralement « les fesses au chand ${ }^{68}$ ».

Une enquête menée pour le ministère de la Défense américaine par des experts de la santé mentale auprès des soldats américains stationnés en Irak confirme la plus grande agressivité des unités proches du champ de bataille ${ }^{69}$. Ainsi, $39 \%$ des interrogés appartenant au corps de la marine (447 personnes interrogées au total) contre $36 \%$ des soldats (total 1320 personnes interrogées au total) estiment dans cette enquête de 2006 que la torture devrait être autorisée pour obtenir des informations. Seuls $38 \%$ des membres de la marine, contre $47 \%$ des autres soldats, croient que les non-combattants devraient être traités avec «dignité et respect 70 ». Ce sont aussi les membres du corps de la marine qui semblent nettement surreprésentés parmi les soldats accusés de crimes de guerre. Ainsi, ce sont des marines qui ont tué vingt-quatre civils dans la ville d'Haditha en 2004 pour « venger » un camarade victime d'un attentat. Les marines sont socialisés dans la manipulation des armes lourdes. Leur devise est "mon fusil est mon meilleur ami. Il est ma vie " "My rifle is my best friend. It is my life »). Ceci traduit un rapport quasi fétichiste avec les armes à feu. Vu sous cet angle, il est peu surprenant que ce soient aussi les membres du corps des marines qui expriment le plus clairement leur frustration de ne pas être capables $\mathrm{de}$ "combattre plus agressivement en raison des restrictions relatives aux règles de l'engagement $71 »$. Certes, depuis l'expérience des «banana wars » (guerres de faible intensité) en Amérique latine (à Cuba, à Puerto Rico, au Honduras, au Nicaragua, au Mexique, en Haïti) le corps des marines est traditionnellement

68. Ainsi s'exprimait un général de l'armée de terre française dans un entretien avec l'auteur.

69. Mental Health Advisory Team (MHAT) UV, Operations Iraqi Freedom 07.07, Final Report, 17 novembre 2006.

70. Ibid.

71. Fainaru S., “For marines, a frustrating fight”, Washington Post, 10 octobre 2004, p. A01. 
plus favorable aux « petites guerres » que les autres unités de l'armée. Il est également vrai que les responsables du corps des marines furent plus hésitants à employer l'artillerie lourde au Vietnam que le chef d'état-major conjoint, le général Westmoreland. Toutefois, seule une petite partie des marines est spécialisée dans les stratégies de contre-insurrection. Mais il convient surtout de souligner que leur stratégie de contre-insurrection met l'accent sur l'ethos combatif. Elle manque de souplesse et privilégie une approche dissuasive. Ainsi, le «mythique » manuel de contre-insurrection Small Wars rédigé par la Marine dans les années 1930 postule que «l'effet moral des chars et des vébicules blindés est probablement plus grand dans des opérations de petite guerre que dans des guerres majeures... des chars sont particulièrement précieux pour contrôler les habitants d'une grande ville $72 »$. En 1993, le diplomate américain Robert Oakley remarque que les marines sont «lourdement armés » en Somalie avec leurs "patrouilles agressives» et impressionnent la population somalienne ${ }^{73}$. En outre, c'est le corps des marines qui cultive le plus un sentiment de spécificité et de supériorité par rapport à la société civile ${ }^{74}$.

L'hypothèse selon laquelle ce sont les unités de combat qui sont le plus prédisposées à des ripostes disproportionnées face à des provocations dans les missions de maintien de l'ordre est aussi confirmée par plusieurs études empiriques. Des enquêtes sur la pacification des populations en Algérie (1957-1958) et en Irlande du Nord (1970-1999) suggèrent que la torture était facilitée par un faible contrôle civil des militaires. Ainsi, le «Bloody Sunday » du 30 janvier 1972 au cours duquel treize personnes ont été tuées aurait été provoqué par un régiment parachutiste qui aurait délibérément tiré sur une foule désarmée. C’est pour cette raison que le gouvernement britannique a décidé d'associer la police civile à la garde des prisons en Irlande ${ }^{75}$. D'autres études démontrent que l'acceptation des opérations de maintien de la paix est généralement négativement corrélée avec l'implication dans des activités spécifiquement guerrières ${ }^{76}$.

Si l'aptitude des unités de combat aux opérations de maintien de l'ordre mérite un examen plus détaillé, on peut citer au moins trois raisons qui les prédisposent à un « usage intense » du recours à la force. La première tient à la formation. Un soldat de combat apprend à manier le fusil, les mitrailleuses ou les grenades et nettement moins les armes non létales comme la matraque ou le gaz lacrymogène. Cette « routine organisationnelle » dans le maniement des armes lourdes explique qu'ils les emploient avec plus de «facilité » et de

72. Cité par Ricks T., Making the Corps, New York, Touchstone, 1998, p. 182.

73. Ibid., p. 183.

74. Ruppert J., One of Us. Officers of Marine, New York, Praeger, 2003, p. 38 et suivantes, p. 97 et suivantes.

75. Voir à ce propos Campbell J.D., "French Algeria and British Northern Ireland. Legitimacy and the rule of law in low intensity conflict", Military Review, mars-avril 2005.

76. Voir à ce propos : Miller L.L., "Do soldiers hate peacekeeping? The case of preventive diplomacy operation in Macedonia", Armed Forces and Society, vol. 23, n³, pp. 415-449. 
«naturel » que les forces policières dans une manifestation. Deuxièmement, la focalisation des unités de combat sur la guerre proprement dite ainsi que sur la « victoire », les prédispose facilement à ignorer les nuances grises de la réalité. Cette vision machiavélique est même fonctionnelle dans l'activité guerrière car elle diminue les inhibitions à tuer autrui. Troisièmement, la préparation permanente à la guerre alimente chez les unités de combat un alarmisme, qui les conduit à surévaluer les dangers de l'environnement ${ }^{77}$. Cette déformation «professionnelle » provenant d'une attention accrue au «worst case » scénario, explique pourquoi il n'est pas rare que certains soldats « combattants » pris de panique, tirent sur des soldats blessés ou sur des enfants.

Notre analyse suggère que l'action des forces américaines en Irak est un exemple typique d'une prophétie auto-réalisatrice. En traitant les populations irakiennes comme des délinquants potentiels, elles ont détruit toute possibilité de construire des identifications positives. Contrairement aux visions simplistes, la lutte des populations irakiennes contre l'occupant américain n'est pas simplement le fait de groupes «terroristes ", mais aussi une lutte contre le mépris qu'une grande partie de la population irakienne attribue à la puissance américaine. Le problème de la spécificité « combative » comme obstacle à la pacification interne est à la fois un problème américain et un phénomène plus « institutionnel ». Une première solution serait une « civilianisation » de l'armée américaine. Il existe bien des armées qui se sentent reconnues par leurs sociétés et qui relativisent leurs spécificités combattantes. Des armées nordiques (Danemark, Suède, Norvège) et celle du Canada, et peut-être même bientôt celles du Japon et de l'Allemagne semblent favorables aux «opérations autres que la guerre » exigeant des compétences polyvalentes. Toutefois, il est peu probable que la «superpuissance » américaine soit prédisposée à relativiser ce qui fait toujours la « substance » d'une armée, c'est-à-dire sa mission de livrer «bataille », et donc son entraînement à la guerre. Mais des responsables qui veulent avoir à leur disposition de vrais «guerriers » devraient tenir compte de l'inadéquation du warrior à un usage plus « subtil » de la force ou à des missions « gendarmiques ». La seule alternative à la " polyvalence » est la création de forces spécialisées dans le maintien de l'ordre. Ces forces deviendraient de facto des "policiers » même si elles appartenaient formellement au ministère de la Défense comme les gendarmes en France.

77. Voir à ce propos Lindemann T., Martin M.L., (dir.), Les Militaires et le recours à la force armée, Paris, l'Harmattan, 2006, introduction. 